\title{
Regulating Surface and Local Chemistry in High Na- content P2-type Cathode to Achieve Ultrahigh Power and Low Temperature Sodium Storage
}

\section{Qinhao Shi}

Shanghai University

Ruijuan Qi

East China Normal University

Xiaochen Feng

Shanghai University

Jing Wang

Yanshan University

Yong Li

Shanghai University

\section{Zhenpeng Yao}

Harvard University https://orcid.org/0000-0001-8286-8257

\section{Xuan Wang}

Shanghai University

Qianqian Li

Shanghai University

Xionggang Lu

Shanghai University

Jiujun Zhang

Shanghai University https://orcid.org/0000-0001-8357-3696

Yufeng Zhao ( $\nabla$ yufengzhao@shu.edu.cn )

Shanghai University

\section{Article}

Keywords: batteries, sodium ion batteries, grid-scale energy storage, sodium storage

Posted Date: September 2nd, 2021

DOI: https://doi.org/10.21203/rs.3.rs-820608/v1 
License: (c) (i) This work is licensed under a Creative Commons Attribution 4.0 International License. Read Full License

Version of Record: A version of this preprint was published at Nature Communications on June 9th, 2022. See the published version at https://doi.org/10.1038/s41467-022-30942-z. 


\section{Regulating Surface and Local Chemistry in High Na-content P2-type Cathode to}

\section{Achieve Ultrahigh Power and Low Temperature Sodium Storage}

Qinhao Shi ${ }^{1, \&}$, Ruijuan $\mathrm{Qi}^{2, \&}$, Xiaochen Feng ${ }^{1, \&}$, Jing Wang ${ }^{3, \&}$, Yong $\mathrm{Li}^{1}$, Zhenpeng Yao ${ }^{4}$, Xuan Wang ${ }^{1}$, Qianqian $\mathrm{Li}^{1}$, Xionggang $\mathrm{Lu}^{1}$, Jiujun Zhang ${ }^{1}$, Yufeng Zhao ${ }^{1 *}$

${ }^{1}$ Institute for Sustainable Energy/College of Sciences, Shanghai University, Shanghai 200444, China

${ }^{2}$ Key Laboratory of Polar Materials and Devices (MOE) and Department of Electronics, East China Normal University, Shanghai 200062, China.

${ }^{3}$ Key Laboratory of Applied Chemistry in Hebei Province, Yanshan University, Qinhuangdao, 066004, China.

${ }^{4}$ Department of Chemistry and Chemical Biology, Harvard University, 12 Oxford Street, Cambridge, Massachusetts 02138, United States

Corresponding email: yufengzhao@shu.edu.cn

${ }^{\&}$ These authors contribute equally to this work.

\section{Abstract}

Application of sodium ion batteries in grid-scale energy storage demands electrode materials that facilitate fast and stable charge storage from room-temperature to subzero temperature range. The key issues that hinder P2-type layered oxides from achieving such goals are their unsatisfied charge transfer kinetics and unavoidable surface fading. Herein, we report a P2-type $\mathrm{Na}_{0.78} \mathrm{Ni}_{0.31} \mathrm{Mn}_{0.67} \mathrm{Nb}_{0.02} \mathrm{O}_{2}$ whereby the trace $\mathrm{Nb}$ substitution simultaneously reduces the electronic band gap and ionic diffusion energy barrier, thus enables fast electron and $\mathrm{Na}^{+}$mobility $\left(\sim 10^{-9} \mathrm{~cm} \mathrm{~s}^{-1}\right.$ at $\left.-40{ }^{\circ} \mathrm{C}\right)$. While the $\mathrm{Nb}$ induced atomic-scale surface pre-construction efficiently prevents the electrolyte penetration and surface metal dissolution. The material demonstrates a record high rate capability $(50 \mathrm{C})$, unprecedented low temperature performance and ultrahigh cycling stability $\left(98 \%\right.$ capacity retention at $-40{ }^{\circ} \mathrm{C}$ with $76 \%$ capacity remaining after 1800 cycles). Different from literatures, this work shows that complete solid-solution is not always critical for high rate performance. 


\section{Introduction}

As an alternative to lithium-ion batteries, sodium-ion batteries (SIBs) have been paid significant attention for grid-scale energy storage due to the greater abundance of sodium sources. Nevertheless, the practical application of SIBs is hindered by the slow $\mathrm{Na}^{+}$transfer kinetics especially at subzero temperatures. Layered transition-metal oxides $\left(\mathrm{Na}_{x} \mathrm{TMO}_{2}, \mathrm{TM}\right.$ represents transition metal) are considered as the most promising cathode families due to their low cost and high theoretical capacities ${ }^{1-11}$. Among the layered structure (mainly P2, O3, according to the count of edge-sharing $\mathrm{TMO}_{6}$ octahedra with the oxygen piling in ABBA or ABCABC stacking ${ }^{12}$ as displayed in Supplementary Fig. 1), $\mathrm{P} 2-\mathrm{Na}_{2 / 3} \mathrm{Mn}_{2 / 3} \mathrm{Ni}_{1 / 3} \mathrm{O}_{2}$, with classic composition has been extensively investigated, which displays a striking specific capacity of $160 \mathrm{mAh} \mathrm{g}^{-1}$ within 2.0-4.5 $\mathrm{V}$ based on $\mathrm{Ni}^{2+/ 3+/ 4+}$ redox couples ${ }^{13-17}$. Nevertheless, such a structure suffers from irreversible structural changes or phase transitions (e.g. P2-O2, P2-OP4) at certain desodiation states ${ }^{17}$ and severe interfacial transition metal dissolutions ${ }^{18-23}$, thus causing wretched rate capability and capacity decay upon cycling.

Efforts have been taken to stabilize the crystal structure during charge/discharge and enable fast $\mathrm{Na}^{+}$(de)intercalation. For instance, proper cations doping (e.g. $\mathrm{Li}^{+}, 18,25$, $\left.\mathrm{Cu}^{2+, 26,27}, \mathrm{Mg}^{2+}, 28,29, \mathrm{Zn}^{2+}{ }^{20}\right)$ are proved to be efficient in eliminating the TM layers gliding and $\mathrm{Na}^{+} /$vacancy ordering, thereby realizing a fast solid-solution reaction. Surface decorations have been applied to improve the surface structural stability upon cycling $23,24,31,32$ while increasing the $\mathrm{Na}$ content was reported to be capable of stabilizing the crystal structure at deep desodiation states. ${ }^{18,33}$ For example, by 
increasing the sodium content to 0.85 , and combining with $\mathrm{Li}^{+}$substitution, a plateaufree P2-type cathode- $\mathrm{Na}_{0.85} \mathrm{Li}_{0.12} \mathrm{Ni}_{0.22} \mathrm{Mn}_{0.66} \mathrm{O}_{2}$ (P2-NLNMO) was developed, which enables a fast $\mathrm{Na}^{+}$mobility $\left(10^{-11}\right.$ to $\left.10^{-10} \mathrm{~cm}^{2} \mathrm{~s}^{-1}\right)$ by realizing the completely solidsolution reaction in the whole experimental potential range. In these studies, sufficient $\mathrm{Na}$ content and complete solid-solution reaction are considered critical to achieving high-rate and highly stable P2-type cathodes. ${ }^{18}$ However, regardless of the great achievements obtained at room temperature, such structures generally encounter a noticeable capacity loss at sub-zero temperatures. ${ }^{34-36}$

In this study, we report a trace $\mathrm{Nb}$-doped high sodium content (0.78) P2-type $\mathrm{Na}_{0.78} \mathrm{Ni}_{0.31} \mathrm{Mn}_{0.67} \mathrm{Nb}_{0.02} \mathrm{O}_{2} \quad(\mathrm{P} 2-\mathrm{NaMNNb})$ with significantly enhanced rate performance and outstanding low temperature (LT) performances. The trace $\mathrm{Nb}$ substitution can simultaneously reduce the electronic band gap and ionic diffusion energy barrier, thus enable fast electron and $\mathrm{Na}^{+}$transfer especially at $\mathrm{Na}$-deficient state. On the other hand, a cation-mixed layer is pre-constructed on the surface of P2$\mathrm{NaMNNb}$ along the direction of $\mathrm{Na}^{+}$diffusion channels at an atomic scale $(3-5 \mathrm{~nm})$, which can inhibit the transition metal dissolution and prevent the electrolyte penetration upon cycling. Note that, the trace $\mathrm{Nb}(0.02)$ substitution enables the relatively high $\mathrm{Ni}$ content $(0.31)$, thus maintains the voltage plateaus of $\mathrm{Ni}^{2+/ 3+/ 4+}$ at $3.7 \sim 3.3 \mathrm{~V}$. Consequently, the P2-NaMNNb materials exhibits an eminent capacity of $96.6 \mathrm{mAh} \mathrm{g}^{-}$ ${ }^{1}$ at $0.5 \mathrm{C}$ with a high working voltage of $3.5 \mathrm{~V}$ (voltage range: $2.4 \sim 4.15 \mathrm{~V}$ ), and an ultrahigh rate capacity $\left(65.8 \mathrm{mAh} \mathrm{g}^{-1} @ 50 \mathrm{C}\right)$. More importantly, the P2-NaMNNb presents almost unchanged diffusion coefficients from 25 to $-40{ }^{\circ} \mathrm{C}\left(\approx 10^{-9} \mathrm{~cm}^{2} \mathrm{~s}^{-1}\right)$, 
which results in a record high capacity retention of $98 \%$ at $-40{ }^{\circ} \mathrm{C}\left(94.5 \mathrm{mAh} \mathrm{g}^{-1} @ 0.5\right.$ C) along with outstanding stability (76\% capacity remaining after 1800 cycles). The full cell assembled with $\mathrm{P} 2-\mathrm{NaMNNb}$ and commercial hard carbons demonstrates a high energy density of $216 \mathrm{Wh} \mathrm{kg}^{-1}$, and extremely high power density of $6.6 \mathrm{~kW} \mathrm{~kg}^{-1}$ (based on the total mass of cathode and anode).

\section{Results and Discussion}

\subsection{Crystal structure of P2-NaMNNb}

The crystal structure of the as-prepared sample was determined by powder X-ray diffraction (XRD) and Rietveld refinement. The refined results of P2-NaMNNb and P2$\mathrm{NaMN}$ ) ( $\left.\mathrm{Na}_{0.78} \mathrm{Ni}_{0.32} \mathrm{Mn}_{0.68} \mathrm{O}_{2}\right)$ fit well with the experimental data (Fig. 1a-b). All the diffraction patterns can be indexed into a hexagonal structure with the space group $P 63 / m m c$, whereby $\mathrm{Mn}, \mathrm{Ni}$ and $\mathrm{Nb}$ atoms are settled on the $2 \mathrm{a}$ site of the transitionmetal layer. There are two kinds of prismatic sites in the $\mathrm{Na}$ layer: $\mathrm{Na}_{\mathrm{f}}$ and $\mathrm{Na}$, sharing two faces or edges with the lower and upper octahedral $\mathrm{TMO}_{6}$, respectively (Supplementary Fig. 1). Similar to previous work $^{33}$, a preferred occupancy at $\mathrm{Na}_{\mathrm{e}}$ sites (Nae: 0.543 mol, Naf: $0.237 \mathrm{~mol}$ ) is observed in $\mathrm{P} 2-\mathrm{NaMNNb}$ to achieve lower electrostatic repulsion between $\mathrm{Na}$ elements. The doping of $\mathrm{Nb}$ causes slight shifts of (002) and (004) peaks to a lower angle (Supplementary Fig. 2), leading to an increase of d-spacing according to the Bragg equation. The elemental ratio of $\mathrm{Na}, \mathrm{Mn}, \mathrm{Ni}$ and $\mathrm{Nb}$ in $\mathrm{P} 2-\mathrm{NaMNNb}$ is determined as $0.78: 0.67: 0.31: 0.02$ by the inductively coupled plasma (ICP) method (Supplementary Table 1). As illustrated in Fig. 1c, the refined crystal structure indicates that $\mathrm{Nb}$ doping can expand the distance between the $\mathrm{TM}$ 
layers from 3.76 to $3.89 \AA$, and the Na-O bond from 2.51 to $2.56 \AA$, which entitles the $\mathrm{Na}^{+}$with enhanced de/intercalation capabilities (Supplementary Tables 2-3).
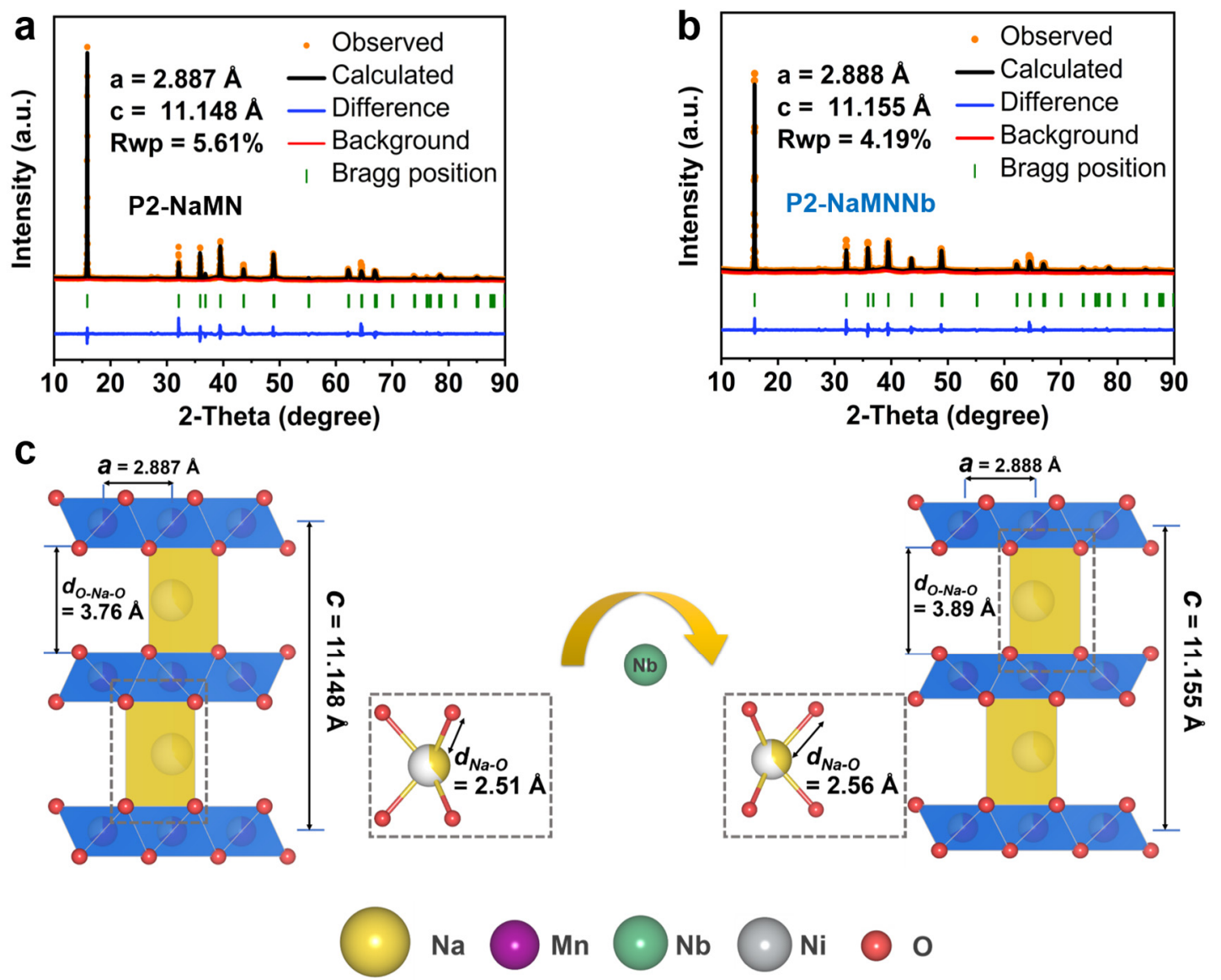

Fig. 1. Structure of the P2-NaMNNb and P2-NaMN cathode material. a, $b$ Rietveld refinements of X-ray diffraction of P2-NaMN and P2-NaMNNb compounds. $\mathbf{c}$ Schematic diagram of the effect of $\mathrm{Nb}$ doping on crystal structure.

\subsection{Atomic structure of P2-NaMNNb}

Aberration-corrected high-angle annular dark-field scanning transmission electron microscopy (HAADF-STEM) was carried out to intensively investigate the effect of $\mathrm{Nb}$ doping on the bulk and surface structure of $\mathrm{P} 2-\mathrm{NaMNNb}$ at an atomic scale (Fig. 2a-b, Supplementary Fig. 3). As the atomic images shown in Fig. 2b-c, it is easy to observe grains projected along [010] zone axis, with the big bright dots for TM atom 
columns, but light elements, such as $\mathrm{Na}$ and $\mathrm{O}$, are invisible, which fits very well with the representatively simulated P2-layered atomic models using the $P 63 / \mathrm{mmc}$ space group as demonstrated by inset. The perfect lattices (without dislocation or defects) illustrate the high crystalline quality of the obtained $\mathrm{P} 2-\mathrm{NaMNNb}$. In addition, indexed from the Fast Fourier transform (FFT) patterns (Supplementary Fig. 4) of the HAADF image in Fig. 2b, the space distance of the lattice fringes for P2-NaMNNb are $0.558 \mathrm{~nm}$ and $0.249 \mathrm{~nm}$ corresponding to (002) and (100) planes (enlarged view of the green rectangle of Fig. 2b), respectively (Fig. 2c). The $c$-axis corresponding to the (002) plane visibly varied when compared with that of P2-NaMN (Supplementary Fig. 7a), which agrees well with the results of XRD refinement. Moreover, as shown in Fig. 2b, it was found that the atom arrangement in the surface region (with a thickness of 3-5 nm) of $\mathrm{P} 2-\mathrm{NaMNNb}$ is different from that of the bulk, which indicates that Nb-doping might induce a pre-constructed surface layer. As reported, such a surface pre-constructed layer is expected to be beneficial to improving structural stability upon $\mathrm{Na}^{+}$de/intercalation during the electrochemical test ${ }^{19,37-40}$. As the enlarged views shown in Fig. 2e-f (corresponding to the red and blue rectangular areas in Fig. 2d), rock-salt-like and spinel-like structures could be observed at the surface and the sub-surface of P2$\mathrm{NaMNNb}$, which may be attributed to the cation disorder arrangement ${ }^{41}$. Furthermore, the evolution of the crystal structure was probed with the assist of line-scan intensity profiles (Fig. 2g-h). As depicted by the simulated lattice structure of pre-constructed layer (Supplementary Fig. 5), the distance between adjacent $\mathrm{Mn}(\mathrm{Ni} / \mathrm{Nb})-\mathrm{I}$ site of the sub-surface is $0.85 \mathrm{~nm}$, which reduced to $0.822 \mathrm{~nm}$ at surface due to the electrostatic 
attraction caused by the transition metals occupying the empty $16 \mathrm{coctahedral} \operatorname{sites}^{42-45}$. Moreover, direct chemical identification (zooming-in area in Fig. 2i) by using the energy dispersive spectrometer (EDS) mappings and elemental line scan analyses further confirm the elemental distribution from bulk to surface, from which we can find that the concentration of $\mathrm{Na}$ at the pre-constructed layer is relatively low but the concentration of $\mathrm{Nb}$ at the same area is relatively high comparing to that at bulk area (Fig. 2i-j, Supplementary Fig. 6). It was supposed that the chemical compositions of the pre-constructed layer for the $\mathrm{Nb}$-doped sample are $\mathrm{Mn}, \mathrm{Ni}, \mathrm{Nb}$ and $\mathrm{O}$.

The above microstructure analyses show that certain contents of $\mathrm{Nb}$ doping into the lattice of P2-NaMN could not only expand d-spacing of $(00 l)$ planes which is beneficial to $\mathrm{Na}^{+}$de/intercalation during cycling, but also form a $\mathrm{Nb}$-rich preconstructed layer (3-5 nm) which might enhance the structure stability of the cathode material. As reported, electron-beam irradiation can provide a feasible stimulus that mimics the effect of electrochemical cycling ${ }^{46}$. Several reports have adopted electronbeam irradiation to estimate the structural stability of the battery materials ${ }^{47,48}$. Here, in situ E-beam irradiation was performed to further investigate the mechanism of $\mathrm{Nb}$ doping on the structure stability enhancement of P2-NaMNNb. Interestingly, we found the $\mathrm{P} 2-\mathrm{NaMNNb}$ and $\mathrm{P} 2-\mathrm{NaMN}$ compounds exhibit different tolerance ability to the electron beam irradiation (Supplementary Fig. 7-8). Under in-situ E beam irradiation, obvious surface reconstruction and subsequent surface amorphous, even material loss can be observed for the P2-NaMN. In contrast, the P2-NaMNNb exhibits impressive tolerability to electron beam irradiation due to the existence of the pre-constructed layer, 
which implies that such a Nb-riched pre-constructed layer might be beneficial to the electrochemical stability of P2-NaMNNb.
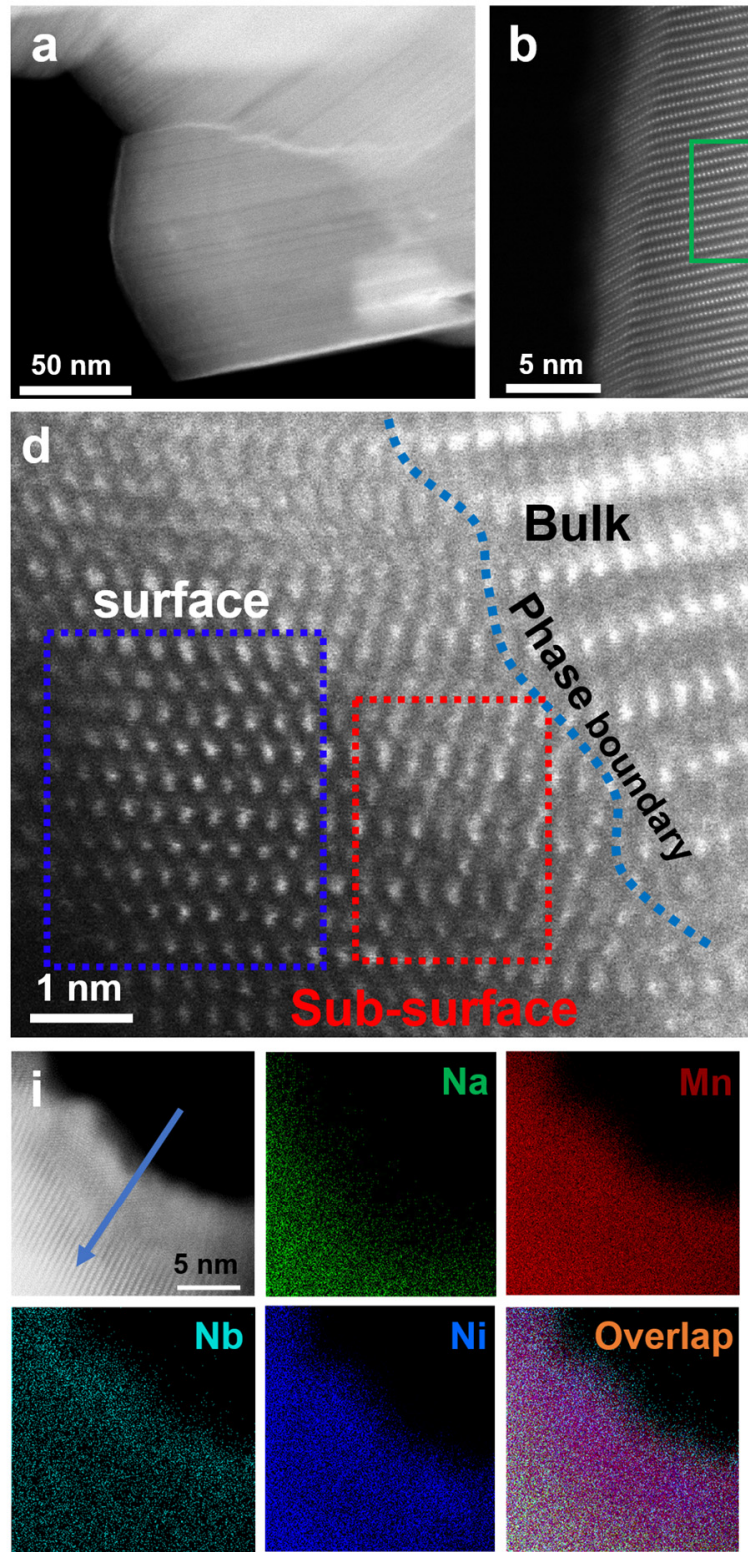
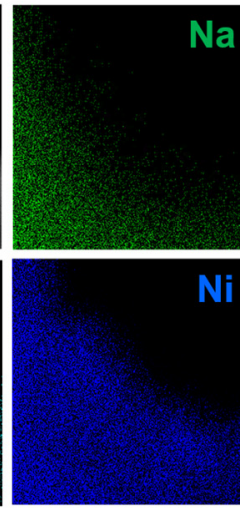

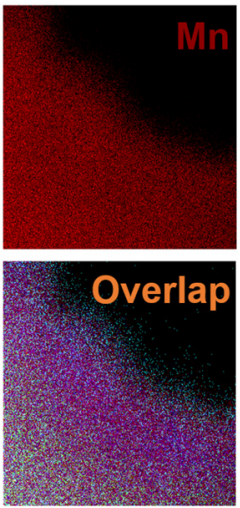

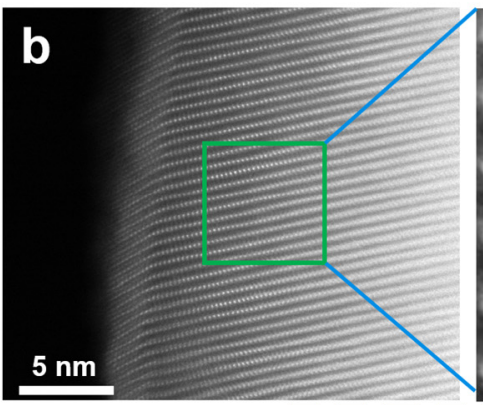
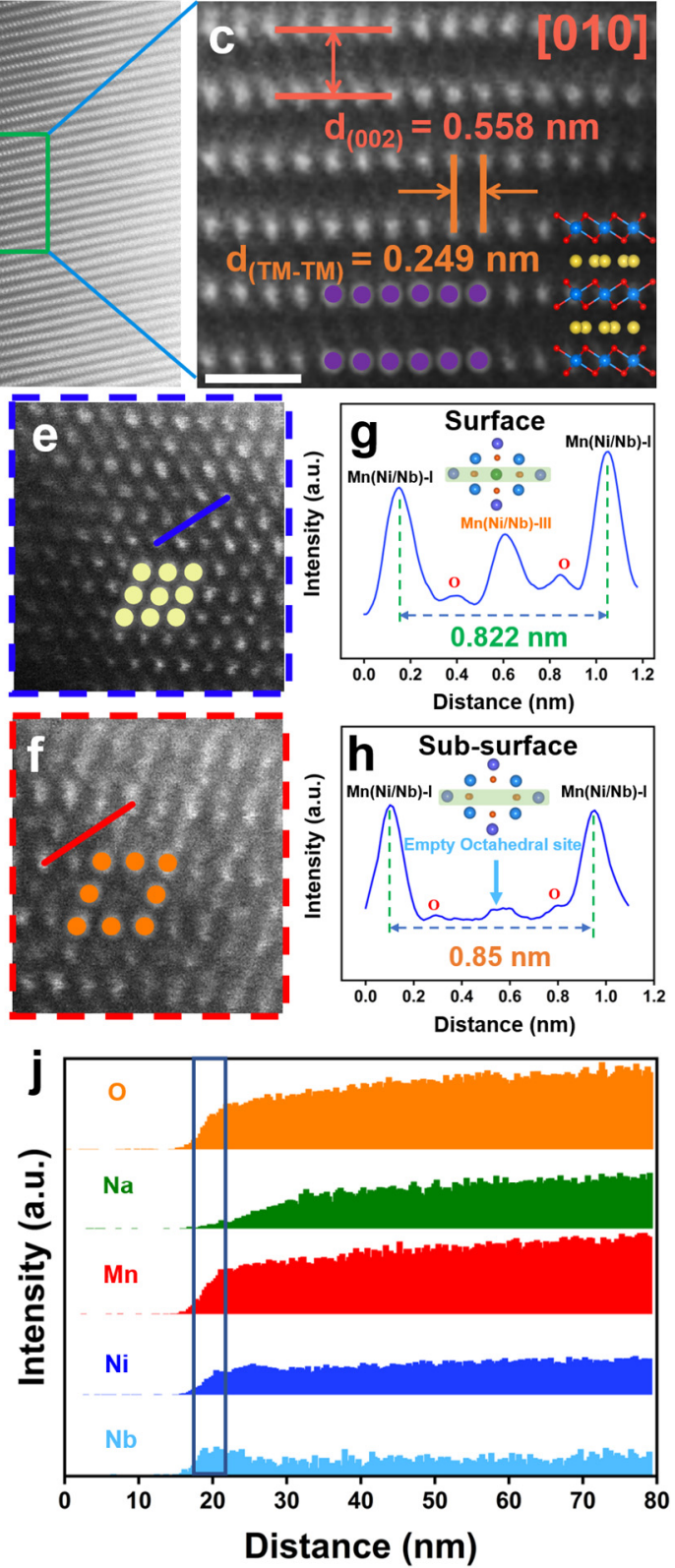

Fig. 2. Atomic structure of the P2-NaMNNb cathode material. a-c STEM-HAADF images of

P2-NaMNNb projected along the [010] zone axis. $\mathbf{d}$ A representative STEM-HAADF image of the interface between pre-constructed layer and bulk phase of P2-NaMNNb. e, f Magnified views of the surface and sub-surface corresponding to the blue and red boxes in Fig. $2 \mathbf{d}$. g, h Line profiles corresponding to the blue line in Fig. 2e and the red line in Fig. 2f. i STEM-EDS elemental maps of 
P2-NaMNNb compounds. $\mathbf{j}$ Energy Spectrum elemental line profiles of from surface to bulk phase corresponding to the blue line in Fig. $2 \mathbf{i}$ and corresponding chemical compositions with O (orange), $\mathrm{Na}$ (green), Mn (red), Ni (blue) and $\mathrm{Nb}$ (cyan-blue).

\subsection{Electrochemical Performance}

The electrochemical performance of $\mathrm{P} 2-\mathrm{NaMNNb}$ and $\mathrm{P} 2-\mathrm{NaMN}$ were consistently tested at room temperature (RT, $\left.25{ }^{\circ} \mathrm{C}\right)$ and LT $\left(-40{ }^{\circ} \mathrm{C}\right)$. Cyclic voltammetry at RT for $\mathrm{P} 2-\mathrm{NaMNNb}$ and $\mathrm{P} 2-\mathrm{NaMN}$ in half-cell systems were tested at a scan rate of $0.2 \mathrm{mV} \mathrm{s}^{-1}$ (Fig. 3a). For P2-NaMNNb, three pairs of redox peaks were found at $3.12 / 3.2,3.28 / 3.34$ and $3.56 / 3.64 \mathrm{~V}$, respectively corresponding to the $\mathrm{Ni}^{2+} / \mathrm{Ni}^{3+} / \mathrm{Ni}^{4+}$ redox couples respectively ${ }^{14-17}$. It is noted that, $\mathrm{Nb}$ doping can efficiently reduce the polarization voltage $(\Delta \mathrm{V})$ between adjacent anodic and cathodic peaks is reduced from 97 to $61 \mathrm{mV}$, which agrees well with the charge/discharge profiles of the P2-NaMNNb and P2-NaMN (Supplementary Fig. 9). The valance states of different elements in the selected potential range were verified through ex-situ XPS characterizations (Supplementary Fig. 10). Galvanostatic charge/discharge curves of $\mathrm{P} 2-\mathrm{NaMNNb}$ from 0.5 to $50 \mathrm{C}$ were tested at $\mathrm{RT}$ to confirm the excellent specific capacity and unprecedented rate capability (Fig. 3b-c). The as prepared $\mathrm{P} 2-\mathrm{NaMNNb}$ demonstrates a high specific capacity of $96.6 \mathrm{mAh} \mathrm{g}^{-1}$ in the voltage range of 2.4-4.15 $\mathrm{V}$ at $0.5 \mathrm{C}\left(1 \mathrm{C}=184 \mathrm{mAh} \mathrm{g}^{-1}\right)$, which remains $65.8 \mathrm{mAh} \mathrm{g}^{-1}$ at $50 \mathrm{C}$. As contrast, the P2-NaMN shows much poorer rate capability, with only $81 \%$ capacity retention $(68.4$ mAh $\left.\mathrm{g}^{-1} @ 10 \mathrm{C}\right)$ of that at $0.5 \mathrm{C}$. The electrochemical performance at LT $\left(-40{ }^{\circ} \mathrm{C}\right)$ of the as prepared samples was also tested to verify the application feasibility at LT (Fig. 
3d-f). Unlike the obvious voltage polarization $(264.7 \mathrm{mV})$ on CV curves for P2-NaMN, P2-NaMNNb performs minor voltage polarization $(126 \mathrm{mV})$ at LT (Fig. 3d). Consequently, the $\mathrm{P} 2-\mathrm{NaMNNb}$ demonstrates a record high capacity retention $(98 \%$ of that at RT) with a discharge capacity of $94.5 \mathrm{mAh} \mathrm{g}^{-1}$ at $0.5 \mathrm{C}$, which maintains 83.6 $\mathrm{mAh} \mathrm{g}^{-1}$ at $5 \mathrm{C}$, and $62.9 \mathrm{mAh} \mathrm{g}^{-1}$ at $10 \mathrm{C}$ (Fig. 3e-f). As contrast, P2-NaMN exhibits an inferior discharge capacity of $69.9 \mathrm{mAh} \mathrm{g}^{-1}$ at $0.5 \mathrm{C}(82 \%$ of that at RT), and delivers 59.7 and 39.2 at $1 \mathrm{C}$ and $2 \mathrm{C}$, respectively. This LT performance of P2-NaMNNb outperforms the cathode materials reported in literature ${ }^{49}$. Note that, the trace $\mathrm{Nb}(0.02)$ substitution enables the relatively high $\mathrm{Ni}$ content $(0.31)$, thus maintains the voltage plateaus of $\mathrm{Ni}^{2+/ 3+/ 4+}$ at 3.7 3.3 $\mathrm{V}$. Therefore, the complete solid-solution reaction is not always the necessities in perusing high rate performance for P2-type layered cathodes. The galvanostatic intermittent titration techniques (GITT) (Fig. 3g-h, Supplementary Fig. 11), as an effective electrochemical test method, were employed to assess $\mathrm{Na}^{+}$transport kinetics of P2-NaMNNb and P2-NaMN at both RT and LT. It is noticeable that, the sodium diffusion coefficient $\left(D_{\mathrm{Na}^{+}}{ }^{+}\right)$of $\mathrm{P} 2-\mathrm{NaMNNb}$ is almost unchanged from $25^{\circ} \mathrm{C}$ to $-40^{\circ} \mathrm{C}$, which is from $10^{-11.36}$ to $10^{-9.09}$ and from $10^{-9.81}$ to $10^{-}$ ${ }^{9.29} \mathrm{~cm}^{2} \mathrm{~s}^{-1}$, respectively. In contrast, the average diffusion coefficients of P2-NaMN are unstable (Supplementary Fig. 11), with $10^{-11.64}$ to $10^{-10.09} \mathrm{~cm}^{2} \mathrm{~s}^{-1}$ at $25^{\circ} \mathrm{C}$, and from $10^{-}$ 11.43 to $10^{-10.06} \mathrm{~cm}^{2} \mathrm{~s}^{-1}$ at $-40{ }^{\circ} \mathrm{C}$. Besides, negligible average ohmic polarization $(3.11$ $\mathrm{mV}$ ) and voltage polarization $(12 \mathrm{mV})$ are achieved for $\mathrm{P} 2-\mathrm{NaMNNb}$ at $25^{\circ} \mathrm{C}$, which is 9.7 and $16.1 \mathrm{mV}$ at $-40{ }^{\circ} \mathrm{C}$ respectively (Fig. 3i), indicating the superior $\mathrm{Na}^{+}$transport kinetics even at extreme low temperature. Subsequently, the long-term stability of P2- 
$\mathrm{NaMNNb}$ was tested under a high rate of $5 \mathrm{C}$ at $25^{\circ} \mathrm{C}$ (Supplementary Fig. 12), with remarkable capacity retention of $80 \%$ after 500 cycles. Promisingly, even cycled under a severe condition of $-40{ }^{\circ} \mathrm{C}$ at $2 \mathrm{C}$-rate, a high reversible discharge capacity of $69 \mathrm{mAh}$ $\mathrm{g}^{-1}(76 \%$ capacity retention) is well kept for $\mathrm{P} 2-\mathrm{NaMNNb}$ after 1800 cycles, with capacity decay rates of $0.013 \%$ per cycle (Fig. 3j, Supplementary Fig. 13). Conversely, P2-NaMN suffered hasty capacity loss in a high rate of $5 \mathrm{C}$ at LT (Fig. 3e), and only maintain the capacity of $77.8 \%$ after 215 cycles at RT (Supplementary Fig. 12). Therefore, the trace $\mathrm{Nb}$ incorporation into the lattice and the resulting surface reconstruction show significant effect in enhancing the electrochemical performance under normal or extreme working conditions. 

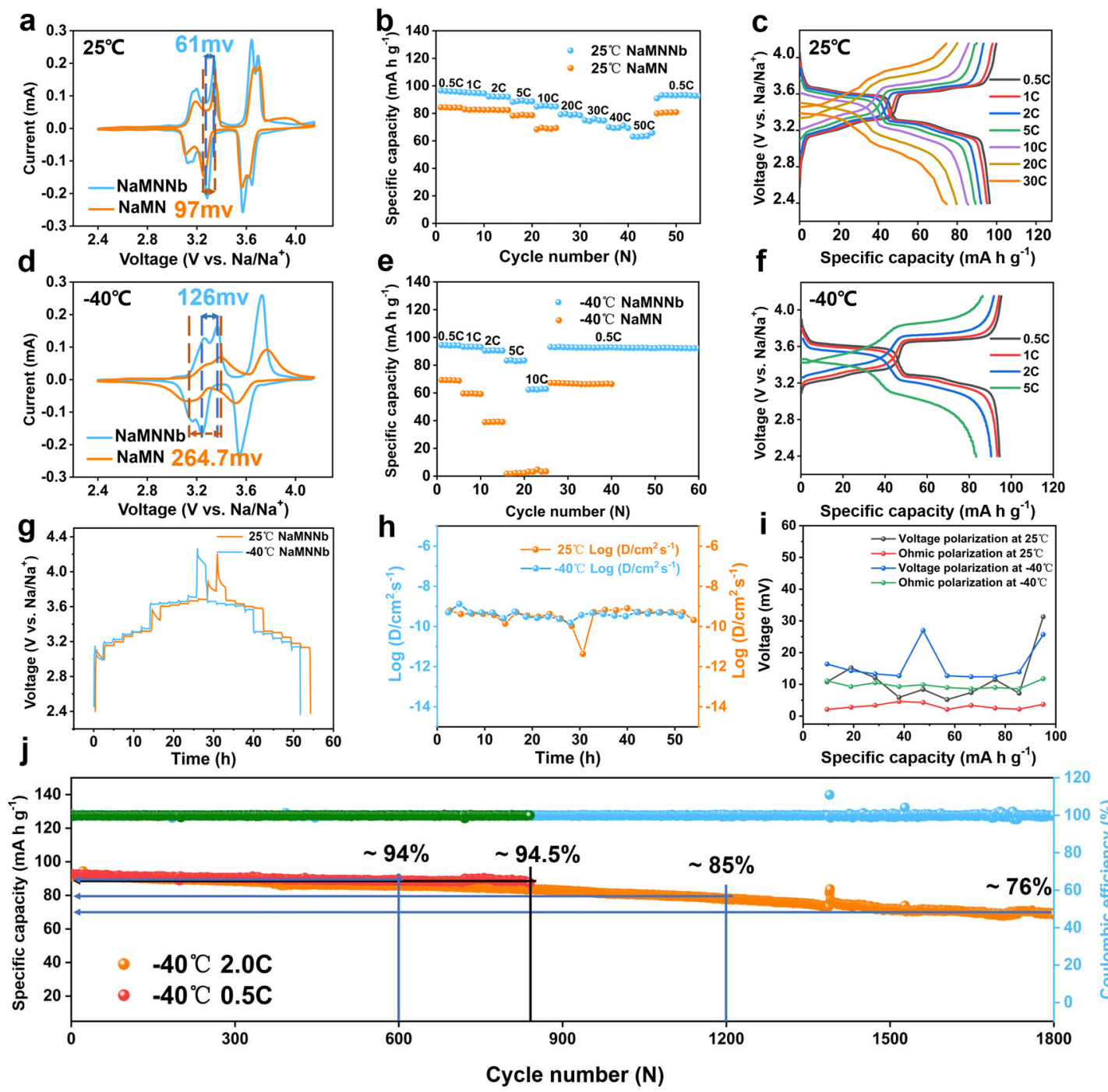

Fig. 3. Electrochemical performance of P2-NaMNNb and P2-NaMN compounds in half cells

in the voltage range of $2.4-4.15 \mathrm{~V}$ at $25^{\circ} \mathrm{C}$ and $-40{ }^{\circ} \mathrm{C}$. a $\mathrm{CV}$ curves of $\mathrm{P} 2-\mathrm{NaMNNb}$ and $\mathrm{P} 2-$

NaMN at a scan rate of $0.2 \mathrm{mV} \mathrm{s}^{-1}$ at $25^{\circ} \mathrm{C} . \mathbf{b}, \mathbf{c}$ rate performance comparison of $\mathrm{P} 2-\mathrm{NaMNNb}$ and $\mathrm{P} 2-\mathrm{NaMN}$ and corresponding charge/discharge curves at $25^{\circ} \mathrm{C}$. d CV curves of $\mathrm{P} 2-\mathrm{NaMNNb}$ and $\mathrm{P} 2-\mathrm{NaMN}$ at a scan rate of $0.2 \mathrm{mV} \mathrm{s}^{-1}$ at $-40{ }^{\circ} \mathrm{C}$. e, f rate performance comparison of P2-NaMNNb and P2-NaMN and corresponding charge/discharge curves at $-40{ }^{\circ} \mathrm{C}$. $\mathrm{g}$ GITT curves of P2-NaMNNb at $25^{\circ} \mathrm{C}$ and $-40{ }^{\circ} \mathrm{C}$. $\mathbf{h}$ The corresponding sodium ion diffusion coefficient $\left(D_{\mathrm{Na}^{+}}{ }^{+}\right)$of $\mathrm{P} 2-\mathrm{NaMNNb}$ calculated from GITT formula at $25{ }^{\circ} \mathrm{C}$ and $-40{ }^{\circ} \mathrm{C}$. i Calculated voltage polarization and ohmic polarization from GITT data during charge process. $\mathbf{j}$ long-term cycling stability at rates of 0.5 and 


\subsection{Structural Evolution}

The structural evolution was inspired for superb electrochemical performance of P2-NaMNNb. In/ex situ XRD patterns were collected during the charge/discharge cycle within the potential range from 2.4 to $4.15 \mathrm{~V}$. It is worth to note that, as $\mathrm{Na}^{+}$ions being extracted during the charging process, the (002) and (004) diffraction peaks sequentially demonstrate explicit shift to lower angles during the charge process due to the increased electrostatic repulsion between the oxygen anion of $\mathrm{Na}$ layers. In the meantime, the (100), (102) and (103) peaks move to higher angles, which may be attributed to the reduction of ionic radius caused by electrochemical oxidation of transition metals. After full cycle, all the characteristic diffraction peaks revert to their original initial position without any new phase observed (Fig. 4a-b, Supplementary Fig. 14), which manifests the distinguishingly reversible chemical reaction of $\mathrm{P} 2-\mathrm{NaMNNb}$ with very small $c$ axis variation $(\sim 2 \%)$, and volume change $(\sim 1.7 \%)$. On the contrary, an obvious peak shows up at $16.4^{\circ}$ for $\mathrm{P} 2-\mathrm{NaMN}$, corresponding to the $\mathrm{P} 2^{\prime}$ phase $(C 2 / c)$, which could cause inferior rate capability and severe capacity decay upon cycling ${ }^{13,28,50,51}$ (Fig. 4b, Supplementary Fig. 15).

On the other hand, the water molecules from electrolytes are easy to react with airinstable NaxTMO 2 by inserting into the Na layer or exchanging $\mathrm{Na}^{+}$with $\mathrm{H}^{+}$, which will form unfavorable hydration phase upon cycling, leading to suppressed $\mathrm{Na}^{+}$ (de)intercalation ability and insufficient electrochemical performances ${ }^{52-55}$. The microstructure evolution after deep cycles was also inspected. Astonishingly, the P2- 
$\mathrm{NaMNNb}$ with pre-constructed cation-mixed layer demonstrates a very small hydration peak at $11.6^{\circ}$ after cycling, which is much stronger for P2-NaMN (Fig. 4b). The HAADF-STEM reveals that the thickness of the surface layer of the P2-NaMNNb remains 3-5 nm after 500 cycles, and the bulk structure remains almost unchanged with $0.558 \mathrm{~nm}$ for the $\mathrm{d}$-spacing of TM-layer and $0.250 \mathrm{~nm}$ for the interlayer spacing of TMatoms (Fig. 4c). As contrast, P2-NaMN encounters a severe surface degradation after 200 cycles (Fig. 4d-e). The elemental information on the Na anode was also examined with energy dispersive x-ray spectroscopy (EDX), whereby the P2-NaMNNb shows negligible TM signals 0.06 at $\%$ and 0.04 at $\%$ for $\mathrm{Mn}$ and $\mathrm{Ni}$, which is 0.3 at $\%$ and 0.25 at $\%$ for $\mathrm{P} 2-\mathrm{NaMN}$ respectively. This indicates that the pre-constructed surface layers can effectively prevent the bulk transition metals from dissolution (Supplementary Fig. 16). Therefore, the pre-constructed layer plays a critical role in inhibiting P2-P2' phase transition and surface degradation, as well as preventing water molecules from entering the crystal lattice, which significantly promote the rate performance and cycling stability of P2-NaMNNb. 

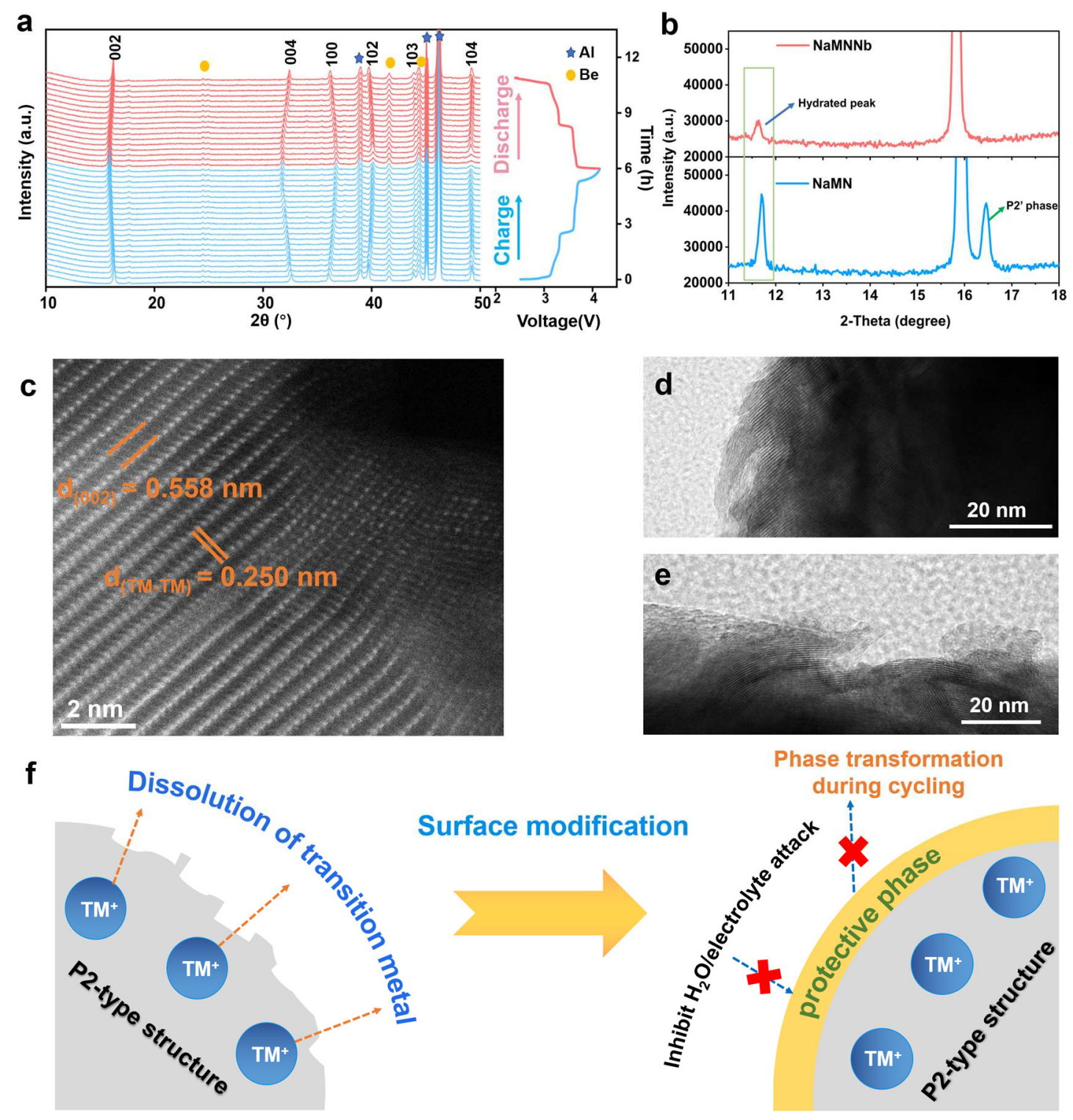

Phase transformation during cycling

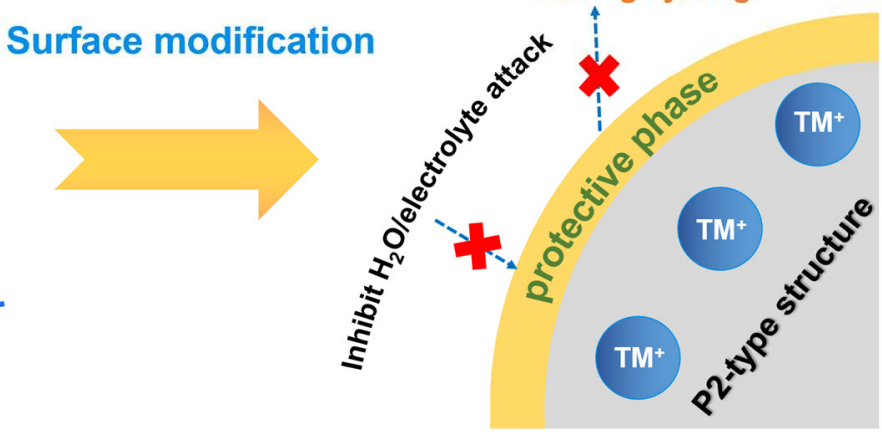

Fig. 4. Structural Evolution a In situ XRD patterns corresponding to the charge and discharge curves between 2.4-4.15 V. b Comparison of (002) diffraction patterns of P2-NaMNNb and P2NaMN after 30 cycles at 0.5 C. c The HAADF-STEM images of the P2-NaMNNb after 500 cycles at 5 C. d, e The TEM images of the P2-NaMN after 200 cycles at 5 C. f Schematic of the protective effect of surface pre-reconstruction on the bulk structure.

\subsection{Density Functional Theory Calculations}

Density functional theory (DFT) calculations were conducted to investigate the (de)intercalation chemistry of $\mathrm{Na}^{+}$in $\mathrm{P} 2-\mathrm{NaMNNb}$. A reasonable $4 \times 4 \times 1 \quad\left(\mathrm{Na}_{24}-\right.$ 
$\left.{ }_{x} \mathrm{Ni}_{10} \mathrm{Mn}_{22} \mathrm{O}_{64}\right)$ supercell with/without $\mathrm{Nb}$ substitution was constructed. After the structural optimization, electronic structure, charge density as well as the $\mathrm{Na}^{+}$migration barrier are calculated. Fig. 5a shows the partial density of states (pDOS) for transition metal $3 \mathrm{~d}$ orbitals and oxygen $2 \mathrm{p}$ orbitals. It is noticed that after $\mathrm{Nb}$ doping, $\mathrm{Mn} \mathrm{e}_{g}$ orbital spilt to two peaks and one of them moves below the Fermi level to maintain charge conservation and reduce the band gap from $0.500 \mathrm{eV}$ to $0.332 \mathrm{eV}$ according to the total density of states, underlining an enhanced electronic conductivity (Fig. 5b-c, Supplementary Fig. 17). ${ }^{57,58}$ The charge density and average bond lengths of TM-O and $\mathrm{Na}-\mathrm{O}$ reflects that the interaction between $\mathrm{TM}$ and $\mathrm{O}$ is more intense (Fig. 5d-e) after $\mathrm{Nb}$ doping ${ }^{59-61}$. The introduction of relatively short $\mathrm{Nb}-\mathrm{O}$ bond results in increasing the bond-length of Na-O but shortening that of TM-O, thus enhancing the bond energy of TM-O to maintain structure stability, which is in well accordance of the XRD refinement. The redox voltage potentials of $\mathrm{P} 2-\mathrm{NaMNNb}$, corresponding to different amount of $\mathrm{Na}$, are also simulated and the results are compared with experimental galvanostatic charge/discharge curves (Fig. 5f). It is seen that the calculated platform (black line) is in good accordance with the realistic blue line. Furthermore, calculated total DFT energies of two kinds of $\mathrm{Na}$ sites in P2-type structure illustrate the energy difference between the $\mathrm{Na}_{\mathrm{e}}$ and $\mathrm{Na}_{\mathrm{f}}$ site can be reduced in the $\mathrm{P} 2-\mathrm{NaMNNb}$ (from 83.6 $\mathrm{meV}$ to $35.6 \mathrm{meV}$ ), implying that the hoping is easier when $\mathrm{Nb}$ is involved (Fig. $5 \mathrm{~g})^{62}$.

To better understand the superb rate capability and LT electrochemical activity of $\mathrm{P} 2-\mathrm{NaMNNb}$, the nudged elastic band (NEB) method is performed to evaluate energy barrier $\left(E_{a}\right)$ for $\mathrm{Na}^{+}$migration. Considering the energy of $\mathrm{Na}_{\mathrm{e}}$ site is lower than $\mathrm{Na}_{\mathrm{f}}$ site 
in $\mathrm{Na}_{\mathrm{x}} \mathrm{TMO}_{2}$, we design the diffusion path from $\mathrm{Na}_{\mathrm{e}}$ to $\mathrm{Na}$, then $\mathrm{Na}_{\mathrm{f}}$ back to $\mathrm{Na}_{\mathrm{e}}$ (Fig. 5h). P2-NaMNNb encounters a small $E_{a}$ of $0.22 \mathrm{eV}$ for $\mathrm{Na}^{+}$diffusion compared to 0.27 $\mathrm{eV}$ of P2-NaMN. Besides, the diffusion coefficient of adjacent $\mathrm{Na}^{+}$sites can be reckoned by the formula of $D=a^{2} v^{*} \exp \left(-E a / k_{B} T\right)$, where $a, v^{*}, E a, k_{B}$ and T stand for the hopping distance between two adjacent sites, hopping frequency $\left(10^{13} \mathrm{~s}^{-1}\right)$, calculated largest barrier via each path, Boltzmann's constant, and the temperature, respectively ${ }^{63}$. The calculated $D_{\mathrm{Na}+}$ of P2-NaMNNb is $1.08 \times 10^{-6}$ and $9.74 \times 10^{-8} \mathrm{~cm}^{2}$ $\mathrm{S}^{-1}$ at RT and LT, respectively, which is one order of magnitude higher than that of P2$\operatorname{NaMN}\left(1.60 \times 10^{-7}\right.$ and $\left.8.59 \times 10^{-9} \mathrm{~cm}^{2} \mathrm{~s}^{-1}\right)$. Thereby, the trace $\mathrm{Nb}$ doping can efficiently boost the reaction kinetics at both RT and LT. In addition, the $E a$ for $\mathrm{Na}^{+}$migration at Na-rich status (with the generated model of one Na vacancy) is also calculated, which is $0.667 \mathrm{eV}$ for P2-NaMNNb, and $0.7 \mathrm{eV}$ for P2-NaMN (Fig. 5i). It is worth to note that, as compared to the Na-rich status, the calculated $D_{\mathrm{Na}}{ }^{+}$is much smaller $(0.667 v s$. $0.22 \mathrm{eV}$ ) at $\mathrm{Na}$ deficient state, corresponding to the high charge/discharge potential at GLD curves. Therefore, controlling the charge potential window within a relatively high voltage range $(2.4-4.15 \mathrm{~V})$ would benefit the rate performance of the electrode material, and meanwhile avoiding the Jahn-teller effect of $\mathrm{Mn}^{3+}$. This finding further unveils the theoretical origin of the ultrahigh rate performance of $\mathrm{P} 2-\mathrm{NaMNNb}$. 
a

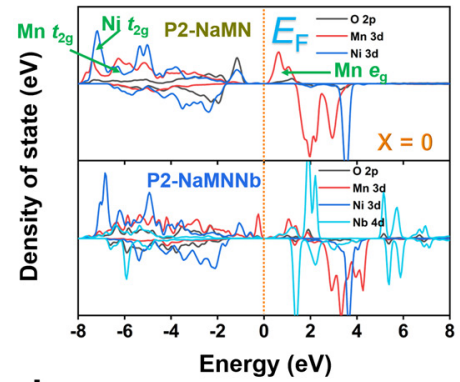

d

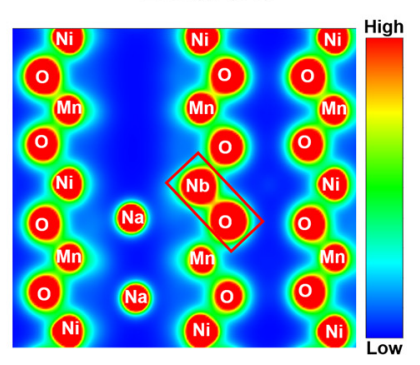

g

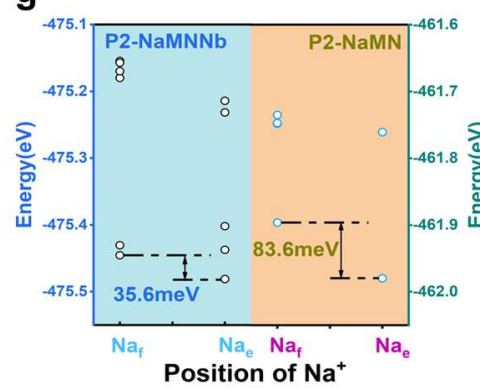

b
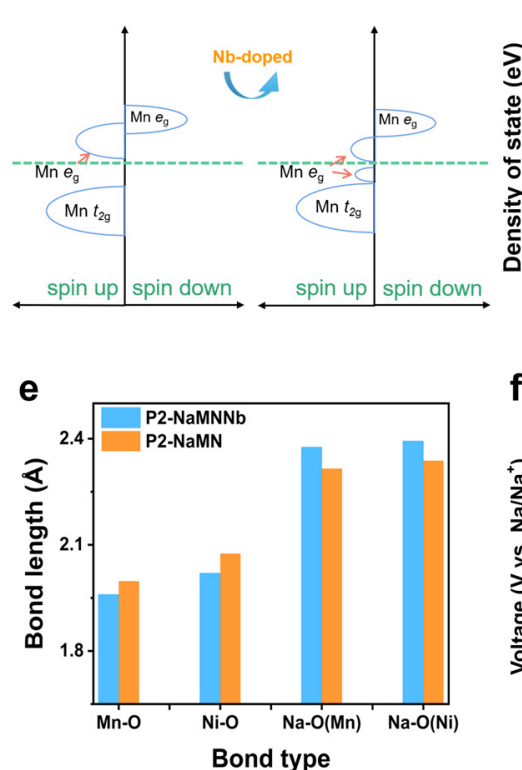

h

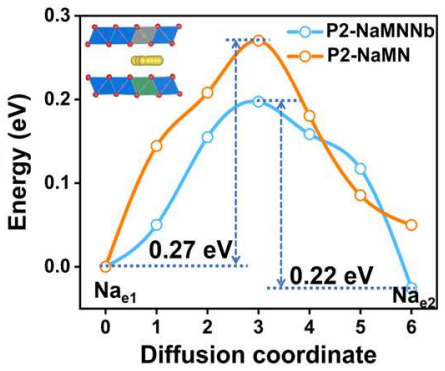

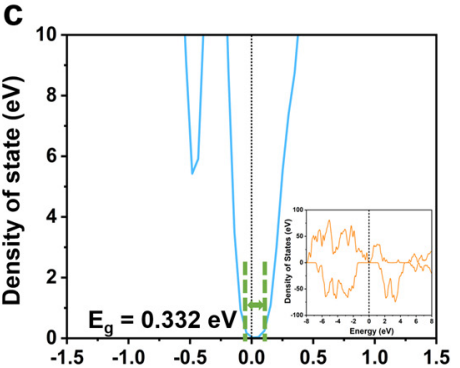

Energy (eV)

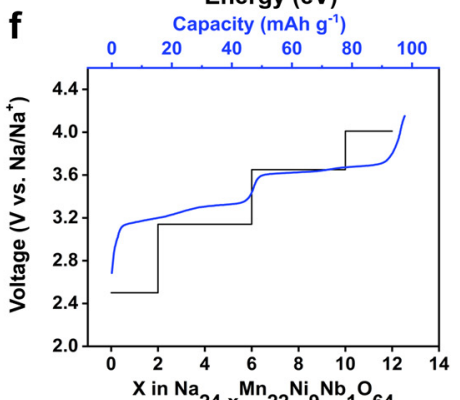

i

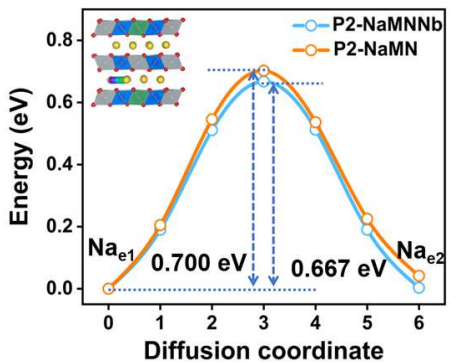

Fig. 5. The evolution of electronic structure. a Partial density of states (pDOS) of O 2p, Mn $3 \mathrm{~d}$, and Ni 3d and Ni 4d orbitals with $x=0$. b Schematic pDOS of P2-NaMN and P2-NaMNNb with $x$ $=0$. $\mathbf{c}$ The total density of state of Na24Ni9Mn22Nb1O64. d Charge density distribution for P2$\mathrm{NaMNNb}$ (visualized by Visualization for Electronic and Structure Analysis (VESTA)), the red regions stand for high charge density and blue regions for low charge density. $\mathbf{e}$ The comparison of average bond length for TM-O and $\mathrm{Na}-\mathrm{O}$ of $\mathrm{P} 2-\mathrm{NaMN}$ and $\mathrm{P} 2-\mathrm{NaMNNb}$. f Comparison of calculated voltage potential and galvanostatic charge/discharge curves. g Calculated energy difference between the Nae and Naf site for $\mathrm{P} 2-\mathrm{NaMNNb}$ (blue background) and $\mathrm{P} 2-\mathrm{NaMN}$ (orange background). h-i Calculated $\mathrm{Na}^{+}$ion diffusion pathways of $\mathrm{P} 2-\mathrm{NaMNNb}$ with single $\mathrm{Na}$ /(one $\mathrm{Na}$ vacancy) and the corresponding $\mathrm{Na}^{+}$migration energy barriers of $\mathrm{P} 2-\mathrm{NaMN}$ and $\mathrm{P} 2-\mathrm{NaMNNb}$. 


\subsection{Full battery constructions}

In order to evaluate practical application potential of $\mathrm{P} 2-\mathrm{NaMNNb}$ cathode in functional condition, a full battery was assembled with the commercial hard carbon as the negative electrode (Fig. 6a). The Na-storage behaviors of the commercial hard carbon anode were examined by galvanostatic charge/discharge and CV tests (Fig. 6b, Supplementary Fig. 18), which delivers a reversible capacity of $280 \mathrm{mAh} \mathrm{g}^{-1}$, with the working potential of $0.11 \mathrm{~V}$ versus $\mathrm{Na}^{+} / \mathrm{Na}$. The full battery delivered a high specific capacity of $96.8 \mathrm{mAh} \mathrm{g}^{-1}$ in the voltage range of $2.3-4.14 \mathrm{~V}$ at $0.5 \mathrm{C}$ at room temperature, corresponding to an energy density of $216 \mathrm{Wh} \mathrm{kg}^{-1}$ (based on the total mass of cathode and anode). The full battery also presents outstanding rate performance, with 94, 91.4, 88, 79.4 and $57.9 \mathrm{mAh} \mathrm{g}^{-1}$ specific capacity at $1 \mathrm{C}, 2 \mathrm{C}, 5 \mathrm{C}, 10 \mathrm{C}, 20 \mathrm{C}$, respectively. Even when tested at $20 \mathrm{C}$, it still demonstrates energy density of $106.2 \mathrm{Wh} \mathrm{kg}^{-1}$, along with a specific power density of $6.6 \mathrm{~kW} \mathrm{~kg}^{-1}$ (Fig. 6c, Supplementary Fig. 19a). Furthermore, the capacity retention of the full battery maintained $81.4 \%$ after 400 cycles in high rate of $5 \mathrm{C}$ (Fig. 6d, Supplementary Fig. 19b). To explore the practical feasibility of the full battery under harsh conditions, the full cell was tested at $-40{ }^{\circ} \mathrm{C}$, which delivers a specific capacity of $94.1 \mathrm{mAh} \mathrm{g}^{-1}$ and $206.8 \mathrm{Wh} \mathrm{kg}^{-1}$ at $0.5 \mathrm{C} \mathrm{(Fig.} \mathrm{6e),}$ with a remarkably high-capacity retention of $97.2 \%$ as compared to that at room temperature, and exhibit $109.7 \mathrm{Wh} \mathrm{kg}^{-1}$ and $1.82 \mathrm{~kW} \mathrm{~kg}^{-1}$, indicating the wondrous ion transport kinetics and reversibility under LT. The cycling durability at LT was also tested at $0.5 \mathrm{C}$, with capacity retention of $85 \%$ after 65 cycles (Fig. 6d, Supplementary Fig. 20). To the best of our knowledge, the low-temperature performance of P2- 
$\mathrm{NaMNNb}$, both in half-cell and full-cell systems, outperforms the state-of-the art cathode materials for SIBs ${ }^{64-67}$ (Fig. 6f, Supplementary Fig. 21, Table 4).
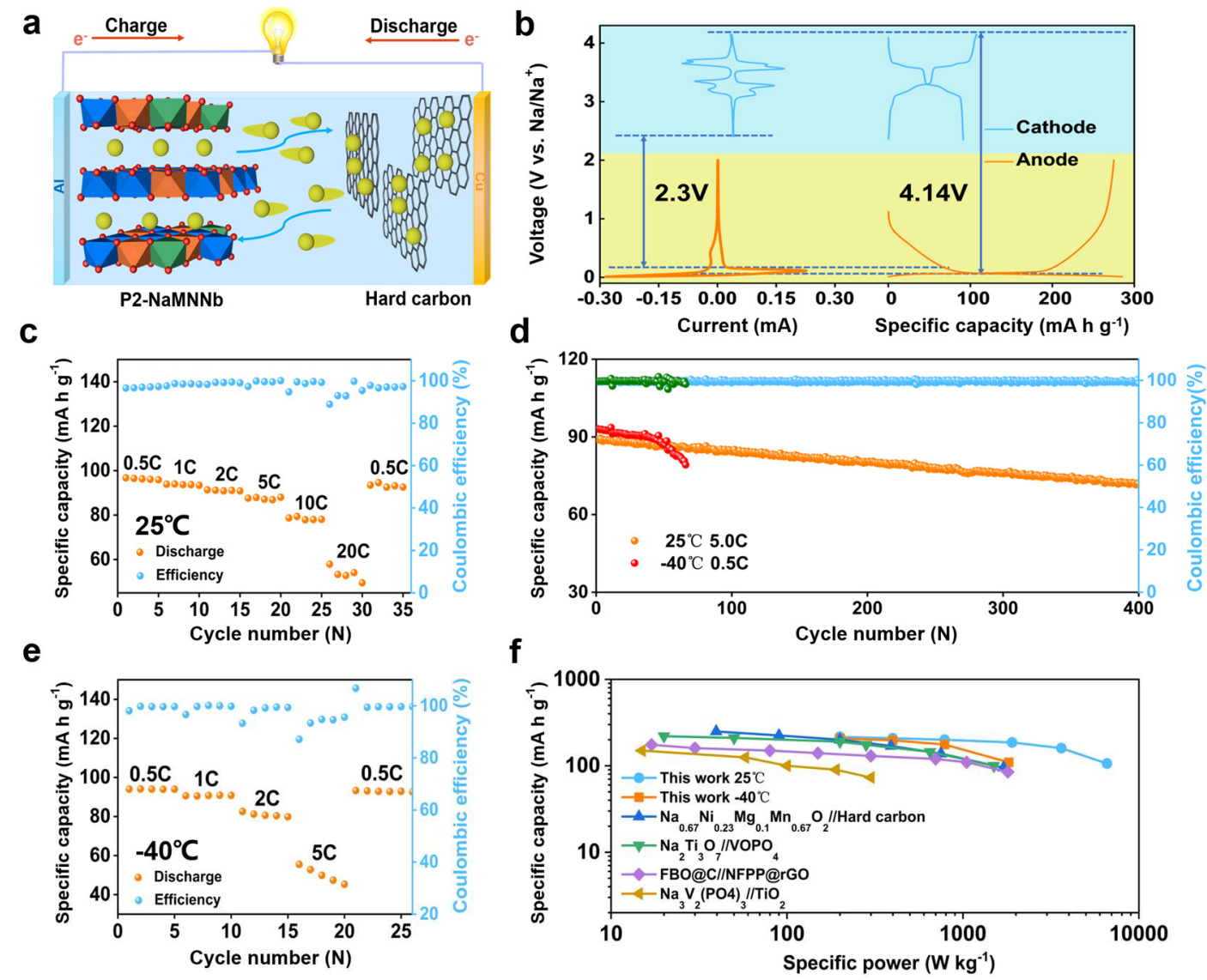

Fig. 6. Electrochemical performance of the full battery at $25^{\circ} \mathrm{C}$ and $-40{ }^{\circ} \mathrm{C}$. a Diagram of the

P2-NaMNNb cathode//hard carbon anode full battery. b Cyclic voltammograms and galvanostatic charge/discharge curves versus specific capacity of $\mathrm{P} 2-\mathrm{NaMNNb}$ and hard carbon electrodes. $\mathbf{c}$ Rate performance of the full battery at $25{ }^{\circ} \mathrm{C}, \mathbf{d}$ Cycling performance in a rate of $5 \mathrm{C}$ at $25{ }^{\circ} \mathrm{C}$. e Rate performance of the full battery at $-40^{\circ} \mathrm{C}$. $\mathbf{f}$ The comparison of the energy/power density with other reported literatures.

\subsection{Discussion}

The superior LT capacity retention and ultrahigh rate performance have been accomplished in the $\mathrm{P} 2-\mathrm{Na}_{0.78} \mathrm{Ni}_{0.31} \mathrm{Mn}_{0.67} \mathrm{Nb}_{0.02} \mathrm{O}_{2}$ materials, which may be attributed 
to the following reasons: I) DFT calculations confirm that the trace $\mathrm{Nb}$ substitution could reduce the electronic band gap and greatly improve the overall conductivity of the material, and meanwhile the $\mathrm{Na}^{+}$diffusion mobility is promoted with suppressed energy barrier especially at Na-deficient status. Therefore both the electronic and ionic mobility can be significantly enhanced, and thus trigger the unprecedented rate performance. II) The doped $\mathrm{Nb}$ induces the formation of an electrochemical corrosionresistant layer in atomic scale, which could prevent $\mathrm{H}_{2} \mathrm{O}$ and solvent molecule enter the crystal lattice, and meanwhile suppress the structural distortion and P2-P2' phase transition, prohibit the loss of transition metals, so that achieve stable $\mathrm{Na}^{+}$ (de)intercalation. III) the trace $\mathrm{Nb}(0.02)$ substitution enables the relatively high $\mathrm{Ni}$ content $(0.31)$, thus maintains the voltage plateaus of $\mathrm{Ni}^{2+/ 3+/ 4+}$ at $3.7 \sim 3.3 \mathrm{~V}$. Therefore, the complete solid-solution reaction is not always the necessities to achieve high rate performance for P2-type layered cathodes. IV) The relatively high $\mathrm{Na}$ content $(0.78)$ enables the crystal structure keep tight at $\mathrm{Na}$ deficient status, which also benefits the fast $\mathrm{Na}^{+}(\mathrm{de})$ intercalation at $\mathrm{Na}$-deficient status.

\section{Conclusions}

In summary, we have successfully constructed a high sodium content P2$\mathrm{Na}_{0.78} \mathrm{Ni}_{0.31} \mathrm{Mn}_{0.67} \mathrm{Nb}_{0.02} \mathrm{O}_{2}$ material with modulated bulk and surface structure. The trace $\mathrm{Nb}$ substitution for $\mathrm{Ni}$ could regulate the bond length of TM-O and $\mathrm{Na}-\mathrm{O}$, and facilitate the high $\mathrm{Na}^{+}$mobility and low activation energy barriers especially at $\mathrm{Na}$ deficient status. Meanwhile, the $\mathrm{Nb}$ doping induced surface preconstruction prevents phase transformation and surface degradation, thus stabilizes $\mathrm{Na}^{+}$(de)intercalation 
reaction during the cycling process. The as obtained material thus demonstrates a record high rate performance reaching the reversible capacity of $65.8 \mathrm{mAh} \mathrm{g}^{-1}$ at $50 \mathrm{C}$ (within 2.4-4.15 V), and excellent LT performance (98\% capacity retention rate up at $-40{ }^{\circ} \mathrm{C}$ ) and remarkable cycling stability $\left(76 \%\right.$ capacity retention after 1800 cycles at $\left.-40{ }^{\circ} \mathrm{C}\right)$. This paper holds a new feasibility to design cathode materials for high rate and LT applications.

\section{Methods}

Computational Methods. DFT calculations were performed with the Vienna ab-initio simulation package (VASP) ${ }^{68}$. The calculations employed the PBE exchange-correlation functional, which is a generalized gradient approximation (GGA) method. The GGA + U method was used because GGA cannot correctly reproduce the localized electronic states of the transition metal oxide materials. The $\mathrm{U}$ values for $\mathrm{Mn}, \mathrm{Ni}, \mathrm{Nb}$ were 3.9, 6.9, $1.5 \mathrm{eV}$. Plane-wave projector augmented wave (PAW) pseudo-potentials were used to represent the core electrons. A plane-wave energy cutoff was $520 \mathrm{eV}$ using for all calculations. NEB calculation is performed to find out the energy barrier for $\mathrm{Na}^{+}$migration ${ }^{69}$. Two model were constructed with single $\mathrm{Na}$ and one vacancy for $\mathrm{Na}^{+}$diffusion to avoid electrostatic repulsion between different $\mathrm{Na}$ ions ${ }^{70}$. All atoms were allowed to relax within the fixed lattice parameters during NEB process. All the calculations were performed on $4 \times 4 \times 1$ supercells of a P2 type $\mathrm{Na}_{24-\mathrm{x}} \mathrm{Ni}_{9} \mathrm{Mn}_{22} \mathrm{Nb}_{1} \mathrm{O}_{64}$ structure.

Materials preparation. The nominal chemical composition of $\mathrm{Na}_{0.78} \mathrm{Ni}_{0.31} \mathrm{Mn}_{0.67} \mathrm{Nb}_{0.02} \mathrm{O}_{2}$ and $\mathrm{Na}_{0.78} \mathrm{Ni}_{0.31} \mathrm{Mn}_{0.67} \mathrm{O}_{2}$ samples were prepared by co-precipitation and the solid phase sintering method. Specifically, the chemicals of Nickel acetate (Aladdin, $>99.0 \%$ ) and manganese acetate (Aladdin, $>99.0 \%$ ) were firstly added into distilled water with a stoichiometric ratio under magnetic 
stirring at $25^{\circ} \mathrm{C}$ for 60 min to obtain a homogeneous solution, marked as A. Weighing $\mathrm{Na}_{2} \mathrm{CO}_{3}$ and dissolve it under magnetic stirring in 50ml deionized water, marked as B. Then, B was dropped into A. The solution was stirred for 12 hours, then washed with water or ethanol for at least 3 times, and dried at $80^{\circ} \mathrm{C}$ in a vacuum oven for 6 hours to obtain the carbonate precursor. Next, mixed the carbonate precursor, $\mathrm{Na}_{2} \mathrm{CO}_{3}$ and $\mathrm{Nb}_{2} \mathrm{O}_{5}$ in to ball mill and milling with high energy for 6 hours. The power was calcined at $500{ }^{\circ} \mathrm{C}$ for 10 hours and then sintered at $900{ }^{\circ} \mathrm{C}$ for 12 hours in Muffle furnace with heating rate of $5{ }^{\circ} \mathrm{C} / \mathrm{min}$ and cooled down to room temperature.

Material characterizations. ICP-AES (PERKINE 7300DV) was used to investigate the composition of elements in samples. X-ray powder diffraction (PXRD) data were performed by utilizing Rigaku smartlab X-ray diffractometer with $\mathrm{Cu}$ K $\alpha$ radiation $(\lambda=1.5418 \AA$ ). In-situ XRD studies, Be piece and Al foil were prepared as X-ray windows with a Swagelok cell during charge and discharge process. Rietveld refinement of X-ray diffraction were analyzed by FullProf Suite. The morphology of all samples are investigated by a scanning electron microscope (SEM). A spherical aberration-corrected (Cs-corrected) scanning transmission electron microscopy (STEM) performed High angle annular dark-field (HAADF) analysis and operated at $300 \mathrm{kV}$ (JEM Grand ARM300F, JEOL).

Electrochemical Measurement. The electrode slurry consisted of active material, acetylene black and polyvinylidene fluoride (PVDF) with the mass ratio of $8: 1: 1$, dissolved in N-Methyl pyrrolidone (NMP), which was coated on an aluminum foil and dried under vacuum at $80^{\circ} \mathrm{C}$ for 12 hours. The coin cells were prepared in an argon-filled glove box. Using sodium tablets as counter electrode and the electrolyte was $1 \mathrm{~mol} \mathrm{~L}^{-1} \mathrm{NaPF} 6$ dissolved in 100\% Diglyme, and the Glass fiber 
(GF/D, Whatman) was used as the separator. All assembled cells were submerged for at least 12 hours before testing the electrochemical performance. Land BT2000 battery system (Wuhan, China) are used to test the charge and discharge process. $\mathrm{D}_{\mathrm{Na}+}$ is calculated by the following formula

$$
\mathrm{D}_{\mathrm{Na}^{+}}=\frac{4}{\pi \tau}\left(\frac{\mathrm{m}_{\mathrm{B}} \mathrm{V}_{\mathrm{M}}}{\mathrm{M}_{\mathrm{B}} \mathrm{S}}\right)^{2}\left(\frac{\Delta \mathrm{E}_{\mathrm{S}}}{\Delta \mathrm{E}_{\tau}}\right)^{2}
$$

Where $\mathrm{D}$ is the diffusion coefficient of $\mathrm{Na}^{+}$in cathode, $\mathrm{V}_{\mathrm{M}}\left(\mathrm{cm}^{3} \mathrm{~mol}^{-1}\right)$ is the molar volume, $\mathrm{m}_{\mathrm{B}}$ and $\mathrm{M}_{\mathrm{B}}$ are molecular weight and relative molar weight of cathode material. $\mathrm{S}\left(1.13 \mathrm{~cm}^{2}\right)$ is the surface area of the electrode. $\tau$ is the time for the applied current during the galvanostatic intermittent titration. $\Delta \mathrm{E}_{\mathrm{S}}$ and $\Delta \mathrm{E}_{\tau}$ are the state of steady voltage and total variation of the battery voltage E during the current pulse.

\section{Data availability}

The data that supporting this work are available from the corresponding author upon reasonable request

\section{Conflicts of interest}

There are no conflicts to declare.

\section{Acknowledgements}

We thank the financial support from the National Natural Science Foundation of China (51774251), Shanghai Science and Technology Commission's "2020 Science and Technology Innovation Action Plan" (20511104003), Shanghai Natural Science Foundation (21ZR1424200), Hebei Natural Science Foundation for Distinguished Young Scholars (B2017203313), Talent Engineering Training Funds of Hebei Province (A201802001). 


\section{Reference}

1. Cao, Y., et al. A New Polyanion Na3Fe2(PO4)P2O7 Cathode with High Electrochemical Performance for Sodium-Ion Batteries. Acs Energy Lett. 5, 3788-3796 (2020).

2. Wang, X., et al. Progress in and application prospects of advanced and cost-effective iron (Fe)based cathode materials for sodium-ion batteries. J. Mater. Chem. A (2021).

3. Shi, S., et al. FeP Quantum Dots Confined in Carbon-Nanotube-Grafted P-Doped Carbon Octahedra for High-Rate Sodium Storage and Full-Cell Applications. Adv. Funct. Mater. 30, 1909283 (2020).

4. Shen, L., et al. Recent Advances and Optimization Strategies on the Electrolytes for Hard Carbon and P-Based Sodium-Ion Batteries. Adv. Funct. Mater. n/a, 2006066

5. Chang, G., et al. A review of phosphorus and phosphides as anode materials for advanced sodium-ion batteries. J. Mater. Chem. A 8, 4996-5048 (2020).

6. Tie, D., et al. Modulating the Interlayer Spacing and Na+/Vacancy Disordering of P2$\mathrm{Na} 0.67 \mathrm{MnO} 2$ for Fast Diffusion and High-Rate Sodium Storage. Acs Appl. Mater. Inter 11, 6978-6985 (2019).

7. Gao, G., et al. Interface-rich mixed $\mathrm{P} 2+\mathrm{T}$ phase NaxCo0.1Mn0.9O2 $(0.44 \leqslant \mathrm{x} \leqslant 0.7)$ toward fast and high capacity sodium storage. J. Mater. Chem. A 6, 6675-6684 (2018).

8. Zhu, Y.-F., et al. Manipulating Layered P2@P3 Integrated Spinel Structure Evolution for HighPerformance Sodium-Ion Batteries. Angew. Chem. Int. Ed. 59, 9299-9304 (2020).

9. Zhao, C., et al. Rational design of layered oxide materials for sodium-ion batteries. Science 370, 708 (2020).

10. Wang, Y., Xiao, R., Hu, Y.-S., Avdeev, M. \&Chen, L. P2-Na0.6[Cr0.6Ti0.4]O2 cationdisordered electrode for high-rate symmetric rechargeable sodium-ion batteries. Nat. Commun. 6, 6954 (2015).

11. Hwang, J.-Y., et al. Radially aligned hierarchical columnar structure as a cathode material for high energy density sodium-ion batteries. Nat. Commun. 6, 6865 (2015).

12. Delmas, C., Fouassier, C. \&Hagenmuller, P. Structural classification and properties of the layered oxides. Physica 99, 81-85 (1980).

13. Liu, Q., et al. P2-type $\mathrm{Na} 2 / 3 \mathrm{Ni} 1 / 3 \mathrm{Mn} 2 / 3 \mathrm{O} 2$ as a cathode material with high-rate and long-life for sodium ion storage. J. Mater. Chem. A 7, 9215-9221 (2019).

14. Liu, K., et al. Insights into the Enhanced Cycle and Rate Performances of the F-Substituted P2-Type Oxide Cathodes for Sodium-Ion Batteries. Adv. Energy Mater. 10, 2000135 (2020).

15. Xiao, Y., et al. A Stable Layered Oxide Cathode Material for High-Performance Sodium-Ion Battery. Adv. Energy Mater. 9, 1803978 (2019).

16. Liu, Z., et al. Ultralow Volume Change of P2-type Layered Oxide Cathode with Controlled Phase Transition by Regulating Distribution of $\mathrm{Na}+$. Angew. Chem. Int. Ed. $\mathbf{n} / \mathbf{a}$,

17. Ma, C., et al. Exploring Oxygen Activity in the High Energy P2-Type Na0.78Ni0.23Mn0.69O2 Cathode Material for Na-Ion Batteries. J. Am. Chem. Soc. 139, 4835-4845 (2017).

18. Jin, T., et al. Realizing Complete Solid-Solution Reaction in High Sodium Content P2-Type Cathode for High-Performance Sodium-Ion Batteries. Angew. Chem. Int. Ed. 59, 14511-14516 (2020). 
19. Guo, S., Li, Q., Liu, P., Chen, M. \&Zhou, H. Environmentally stable interface of layered oxide cathodes for sodium-ion batteries. Nat. Commun. 8, 135 (2017).

20. You, Y., et al. Insights into the Improved High-Voltage Performance of Li-Incorporated Layered Oxide Cathodes for Sodium-Ion Batteries. Chem 4, 2124-2139 (2018).

21. Li, Y., et al. Iron migration and oxygen oxidation during sodium extraction from $\mathrm{NaFeO}$. Nano Energy 47, 519-526 (2018).

22. Wang, Q.-C., et al. Tuning P2-Structured Cathode Material by Na-Site Mg Substitution for NaIon Batteries. J. Am. Chem. Soc. 141, 840-848 (2019).

23. Jo, J. H., et al. Sodium-Ion Batteries: Building Effective Layered Cathode Materials with LongTerm Cycling by Modifying the Surface via Sodium Phosphate. Adv. Funct. Mater. 28, 1705968 (2018).

24. Jo, C.-H., et al. Bioinspired Surface Layer for the Cathode Material of High-Energy-Density Sodium-Ion Batteries. Adv. Energy Mater. 8, 1702942 (2018).

25. Yang, L., et al. Lithium-Doping Stabilized High-Performance P2Na0.66Li0.18Fe0.12Mn0.7O2 Cathode for Sodium Ion Batteries. J. Am. Chem. Soc. 141, 6680-6689 (2019).

26. Xu, S., et al. Suppressing the voltage decay of low-cost P2-type iron-based cathode materials for sodium-ion batteries. J. Mater. Chem. A 6, 20795-20803 (2018).

27. Yan, Z., et al. A Hydrostable Cathode Material Based on the Layered P2@P3 Composite that Shows Redox Behavior for Copper in High-Rate and Long-Cycling Sodium-Ion Batteries. Angew. Chem. Int. Ed. 58, 1412-1416 (2019).

28. Wang, P.-F., et al. Suppressing the P2-O2 Phase Transition of $\mathrm{Na} 0.67 \mathrm{Mn} 0.67 \mathrm{Ni} 0.33 \mathrm{O} 2$ by Magnesium Substitution for Improved Sodium-Ion Batteries. Angew. Chem. Int. Ed. 55, 74457449 (2016).

29. Singh, G., et al. High Voltage Mg-Doped Na0.67Ni0.3-xMgxMn0.7O2 ( $\mathrm{x}=0.05,0.1)$ Na-Ion Cathodes with Enhanced Stability and Rate Capability. Chem. Mater. 28, 5087-5094 (2016).

30. Wang, Y., et al. Ultralow-Strain Zn-Substituted Layered Oxide Cathode with Suppressed P2O2 Transition for Stable Sodium Ion Storage. Adv. Funct. Mater. 30, 1910327 (2020).

31. Dang, R., et al. Na+-Conductive Na2Ti3O7-Modified P2-type Na2/3Ni1/3Mn2/3O2 via a Smart in Situ Coating Approach: Suppressing Na+/Vacancy Ordering and P2-O2 Phase Transition. Acs Appl. Mater. Inter 11, 856-864 (2019).

32. Alvarado, J., et al. Improvement of the Cathode Electrolyte Interphase on P2$\mathrm{Na} 2 / 3 \mathrm{Ni} 1 / 3 \mathrm{Mn} 2 / 3 \mathrm{O} 2$ by Atomic Layer Deposition. Acs Appl. Mater. Inter 9, 26518-26530 (2017).

33. Zhao, C., et al. Revealing High Na-Content P2-Type Layered Oxides as Advanced Sodium-Ion Cathodes. J. Am. Chem. Soc. 142, 5742-5750 (2020).

34. Li, Y., et al. Construction nasicon-type NaTi2(PO4)3 nanoshell on the surface of P2-type $\mathrm{Na} 0.67 \mathrm{Co} 0.2 \mathrm{Mn} 0.8 \mathrm{O} 2$ cathode for superior room/low-temperature sodium storage. Chem. Eng. J. 402, 126181 (2020).

35. Wang, X., et al. Rational design of $\mathrm{Na} 0.67 \mathrm{Ni} 0.2 \mathrm{Co} 0.2 \mathrm{Mn} 0.6 \mathrm{O} 2$ microsphere cathode material for stable and low temperature sodium ion storage. Chem. Eng. J. 428, 130990 (2022).

36. Li, Y., et al. A durable P2-type layered oxide cathode with superior low-temperature performance for sodium-ion batteries. SCIENCE CHINA Materials

37. Zhao, Y., et al. Surface Structural Transition Induced by Gradient Polyanion-Doping in Li-Rich 
Layered Oxides: Implications for Enhanced Electrochemical Performance. Adv. Funct. Mater. 26, 4760-4767 (2016).

38. Ahn, J., et al. Nanoscale Zirconium-Abundant Surface Layers on Lithium- and ManganeseRich Layered Oxides for High-Rate Lithium-Ion Batteries. Nano Lett. 17, 7869-7877 (2017).

39. Liu, S., et al. Surface Doping to Enhance Structural Integrity and Performance of Li-Rich Layered Oxide. Adv. Energy Mater. 8, 1802105 (2018).

40. Guo, S., et al. Cation-mixing stabilized layered oxide cathodes for sodium-ion batteries. Sci. Bull 63, 376-384 (2018).

41. Li, X., et al. Fundamental Insight into Zr Modification of Li- and Mn-Rich Cathodes: Combined Transmission Electron Microscopy and Electrochemical Impedance Spectroscopy Study. Chem. Mater. 30, 2566-2573 (2018).

42. Tang, D., et al. Surface Structure Evolution of LiMn2O4 Cathode Material upon Charge/Discharge. Chem. Mater. 26, 3535-3543 (2014).

43. Amos, C. D., Roldan, M. A., Varela, M., Goodenough, J. B. \&Ferreira, P. J. Revealing the Reconstructed Surface of Li[Mn2]O4. Nano Lett. 16, 2899-2906 (2016).

44. Chen, B., Ben, L., Yu, H., Chen, Y. \&Huang, X. Understanding Surface Structural Stabilization of the High-Temperature and High-Voltage Cycling Performance of Al3+-Modified LiMn2O4 Cathode Material. Acs Appl. Mater. Inter 10, 550-559 (2018).

45. Lee, S., et al. Hierarchical Surface Atomic Structure of a Manganese-Based Spinel Cathode for Lithium-Ion Batteries. Angew. Chem. Int. Ed. 54, 1153-1158 (2015).

46. Zou, L., et al. Unlocking the passivation nature of the cathode-air interfacial reactions in lithium ion batteries. Nat. Commun. 11, 3204 (2020).

47. Li, X., et al. Robust Surface Reconstruction Induced by Subsurface Ni/Li Antisites in Ni-Rich Cathodes. Adv. Funct. Mater. n/a, 2010291

48. Zhan, C., Wu, T., Lu, J. \&Amine, K. Dissolution, migration, and deposition of transition metal ions in Li-ion batteries exemplified by Mn-based cathodes - a critical review. Energy Environ. Sci. 11, 243-257 (2018).

49. Yang, Q., et al. Advanced P2-Na2/3Ni1/3Mn7/12Fe1/12O2 Cathode Material with Suppressed P2-O2 Phase Transition toward High-Performance Sodium-Ion Battery. Acs Appl. Mater. Inter 10, 34272-34282 (2018).

50. Hou, P., Li, F., Wang, Y., Yin, J. \&Xu, X. Mitigating the P2-O2 phase transition of high-voltage $\mathrm{P} 2-\mathrm{Na} 2 / 3[\mathrm{Ni1} / 3 \mathrm{Mn} 2 / 3] \mathrm{O} 2$ cathodes by cobalt gradient substitution for high-rate sodium-ion batteries. J. Mater. Chem. A 7, 4705-4713 (2019).

51. Huang, Y., et al. Vitalization of $\mathrm{P} 2-\mathrm{Na} 2 / 3 \mathrm{Ni} 1 / 3 \mathrm{Mn} 2 / 3 \mathrm{O} 2$ at high-voltage cyclability via combined structural modulation for sodium-ion batteries. Energy Storage Mater. 29, 182-189 (2020).

52. Boyd, S., Dhall, R., LeBeau, James M. \&Augustyn, V. Charge storage mechanism and degradation of P2-type sodium transition metal oxides in aqueous electrolytes. J. Mater. Chem. A 6, 22266-22276 (2018).

53. Zhang, Y., Zhang, R. \&Huang, Y. Air-Stable NaxTMO2 Cathodes for Sodium Storage. Front Chem 7, (2019).

54. Duffort, V., Talaie, E., Black, R. \&Nazar, L. F. Uptake of $\mathrm{CO} 2$ in Layered P2$\mathrm{Na} 0.67 \mathrm{Mn} 0.5 \mathrm{Fe} 0.5 \mathrm{O} 2$ : Insertion of Carbonate Anions. Chem. Mater. 27, 2515-2524 (2015).

55. Han, M. H., et al. Moisture exposed layered oxide electrodes as Na-ion battery cathodes. $J$. 
Mater. Chem. A 4, 18963-18975 (2016).

56. Hao, X., et al. Charge, Orbital, and Magnetic Ordering in YBaFe2O5 from First-Principles Calculations. Inorg. Chem. 47, 4734-4739 (2008).

57. Kim, H. J., et al. Controlled Oxygen Redox for Excellent Power Capability in Layered SodiumBased Compounds. Adv. Energy Mater. 9, 1901181 (2019).

58. Shi, S., Ouyang, C., Lei, M. \&Tang, W. Effect of Mg-doping on the structural and electronic properties of LiCoO2: A first-principles investigation. J. Power Sources 171, 908-912 (2007).

59. Wang, C., et al. Tuning local chemistry of P2 layered-oxide cathode for high energy and long cycles of sodium-ion battery. Nat. Commun. 12, 2256 (2021).

60. Yu, H., et al. Study of the lithium/nickel ions exchange in the layered LiNi0.42Mn0.42Co0.16O2 cathode material for lithium ion batteries: experimental and firstprinciples calculations. Energy Environ. Sci. 7, 1068-1078 (2014).

61. Pearce, P. E., et al. Evidence for anionic redox activity in a tridimensional-ordered Li-rich positive electrode $\beta$-Li2IrO3. Nat.Mater. 16, 580-586 (2017).

62. Wang, P.-F., et al. Na\&lt;sup\&gt;+\&lt;/sup\&gt;/vacancy disordering promises high-rate Naion batteries. Sci. Adv. 4, eaar6018 (2018).

63. Rui, X., et al. A Low-Temperature Sodium-Ion Full Battery: Superb Kinetics and Cycling Stability. Adv. Funct. Mater. 31, 2009458 (2021).

64. Peng, B., Sun, Z., Zhao, L., Li, J. \&Zhang, G. Dual-Manipulation on P2$\mathrm{Na} 0.67 \mathrm{Ni0} .33 \mathrm{Mn} 0.67 \mathrm{O} 2$ Layered Cathode toward Sodium-Ion Full Cell with Record Operating Voltage Beyond 3.5 V. Energy Storage Mater. 35, 620-629 (2021).

65. Li, H., et al. An advanced high-energy sodium ion full battery based on nanostructured Na2Ti3O7/VOPO4 layered materials. Energy Environ. Sci. 9, 3399-3405 (2016).

66. Cao, Y., et al. All-Climate Iron-Based Sodium-Ion Full Cell for Energy Storage. Adv. Funct. Mater. $\mathbf{n} / \mathbf{a}, 2102856$

67. Fang, Y., et al. MXene-Derived Defect-Rich TiO2@rGO as High-Rate Anodes for Full Na Ion Batteries and Capacitors. Nano-Micro Letters 12, 128 (2020).

68. Kresse, G. \&Furthmüller, J. Efficient iterative schemes for ab initio total-energy calculations using a plane-wave basis set. Physical Review B 54, 11169-11186 (1996).

69. Henkelman, G., Uberuaga, B. P. \& Jónsson, H. A climbing image nudged elastic band method for finding saddle points and minimum energy paths. J. Chem. Phys. 113, 9901-9904 (2000).

70. Park, Y. J., et al. A New Strategy to Build a High-Performance $\mathrm{P}^{\prime}$ 2-Type Cathode Material through Titanium Doping for Sodium-Ion Batteries. Adv. Funct. Mater. 29, 1901912 (2019). 


\section{Supplementary Files}

This is a list of supplementary files associated with this preprint. Click to download.

- NCNbSCISupplementaryInformation815.docx 